\title{
Toward integrative annotation of cell type-specific epigenomes to better understand human biology and disease
}

\author{
"The reported findings support the idea that epigenetic \\ heterogeneity between individuals is one of the major mechanisms to \\ sustain cell-to-cell variability."
}

Keywords: chromatin $\bullet$ enhancers $\bullet$ epigenome $\bullet$ histone modifications

An important breakthrough in large-scale mapping studies of the human epigenome has brought us closer to better understanding of gene expression responses to environmental signals. The ENCODE Project dissected and cataloged a vast array of regulatory elements in human cells, and the NIH Roadmap Epigenomics Mapping Consortium produced the reference epigenomes for 127 tissues and cell types [1,2]. These biological samples encompass embryonic and adult tissues from healthy individuals as well as from patients with different disorders. The NIH-supported initiative established global genomic connections, disease variants and epigenomic marks of relevant cell types for diverse human traits. Collectively, these studies demonstrated the central role of epigenomic processes for understanding gene regulation, cellular differentiation and human disease.

Although the genome in all the cells in a human body is essentially the same, the epigenetic signatures show significant differences in cell types of different lineages. Approximately 20,000-40,000 enhancers provide critical information directing cell-type-specific transcription as being regulated by the coordination of cell- and signal-dependent transcription factors (TFs), cofactors and histone modifications [1].

TFs bind to genomic sequences known as hotspots through mediated by combinations of core and stage-specific factors and other chromatin-associated proteins [3,4]. In the early phase of adipogenesis, approximately 12,000 hotspots can recruit TFs both simultaneously and sequentially to facilitate lineage commitment. It was demonstrated that hotspots are further integrated into super enhancers, large genomic domains $[5,6]$. The cell type-specific super-enhancers are defined by a set of specific TFs and enriched by distinct epigenomic modifications to integrate external stimuli on the genome in cooperative fashion [7].

The study reported by Tsankov et al. [8] analyzed genome-wide binding data for 38 TFs during human embryonic stem cell (ESC) differentiation into the three germ layers. They documented the lineage-specific behavior of TFs; in addition to the ability to modulate the chromatin landscape, the binding of TFs to enhancer regions is accompanied with specific loss of DNA methylation in one germ layer, and a reciprocal gain in the other layers. Liu et al. [9] dissected further the enhancer composition by demonstrating that a large 1-2 MDa complex composed of a diverse set of TFs is capable to assemble at the estrogen-responsive enhancers. This type of enhancers, also known as mega transcription factor-bound in transenhancers or the MegaTrans complex, is enriched at the inducible genomic hotspots that are characterized by recruitment of p300, mediator and DNA-dependent protein kinase.

Distant chromatin interactions are essential for creating the 3D nuclear scaffold for facilitating enhancer-promoter communication in a cell-specific fashion. It was estimated that over one million regulatory elements are in the mammalian genome for supporting distant enhancer-promoter interactions in a cell- or tissue-specific manner [10,11]. The new research revealed insights into genome
Epigenomics
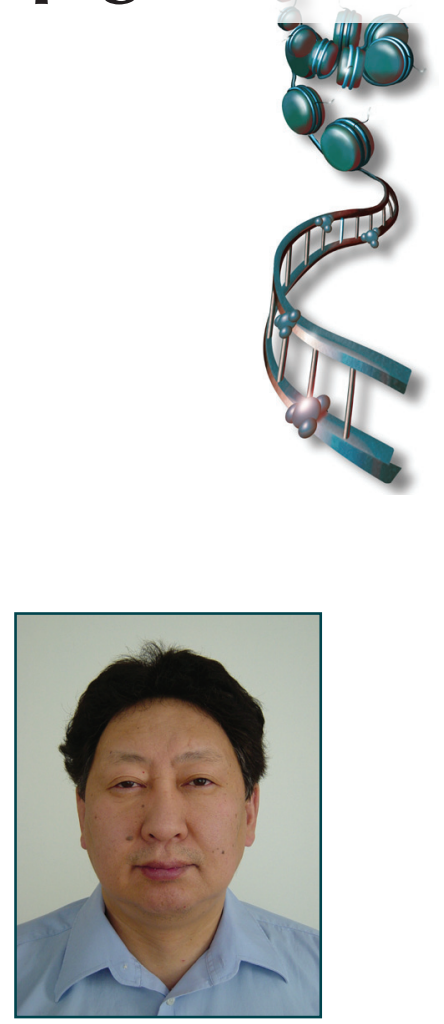

Dashzeveg

Bayarsaihan

Center for Regenerative

Medicine \& Skeletal

Development, Department

of Reconstructive

Sciences, University of

Connecticut Health Center,

262 Farmington Avenue,

Farmington, CT 06030, USA

Tel.: +1 8606795456

Fax: +1 8606792910

dashzeveg@uchc.edu 
topology and its role in gene expression [12]. In mouse ESCs and fetal liver cells, active interacting elements are enriched in active histone modification marks with higher TF occupancy, whereas nonactive distal regulatory elements are marked with repressive histone modifications. Furthermore, nonrandom clustering of genes that are coregulated in spatial nuclear space correlates with their expression and biological role. In ESCs, the strongest clustering occurs between genes encoding TFs that control key developmental processes [12]. Therefore, higher-order genomic architecture is emerging as an important regulator of gene expression.

\section{"An important breakthrough in large-scale mapping studies of the human epigenome has brought us closer to better understanding of gene expression responses to environmental signals."}

Chromosomes are divided into topology activation domains (TADs), architectural building blocks that provide a framework for the modular organization of chromosomes [13]. In human lymphoblastoid cells, chromatin is structured into 10,000 loop domains that carry distinct histone modifications [14]. The structure created by chromosome loops is conserved across cell types and species providing scaffolding for enhancer-promoter interactions. In ESCs, enhancerpromoter cross talks occur within chromosome loops flanked by two interacting CTCF [14,15]. This type of insulated chromatin organization is important for proper coregulation of lineage-specific developmental genes. Furthermore, the modular organization is well preserved in conserved syntenic regions between the genomes of different mammalian species [16]. Although CTCF defines the borders of TADs, divergent CTCF binding between species is correlated with species-specific differences of internal subdomain structures, which is a subject of mutation-driven evolutionary changes that could affect the CTCF recruitment. CTCF often co-occupies genomic regions with cohesin to mediate $3 \mathrm{D}$ chromosome looping [15]. The reduced dosage of cohesin alters the genome architecture of the regenerating islet-derived gene cluster and decreases their expression in the pancreas of mice heterozygous for SA1, one of the cohesin subunits [17].

Although a number of reports documented chromatin interaction dynamics, the full spectrum of genomewide connections during embryonic development and lineage commitment is still uncharted territory. Chromatin connectivity mapping in human ESCs and four human ESC-derived lineages uncovered dynamic chromatin reorganization during lineage commitment [18]. Interestingly, although overall domain topology stays relatively stable, interactions within a topology domain and between TADs change significantly during ESC differentiation.

Mutations at the distal sites could contribute to the dysregulation of oncogenes via long-range chromatin interactions. Babaei et al. [19] found that viral cancercausing insertions associate with genomic hotspots that are significantly enriched for TF-binding sites. These findings support the importance of considering 3D genomic interactions as potential targets for therapeutic intervention in human diseases.

In another study, Amin et al. [20] reported epigenetic control mechanisms for tissue-specific expression of lincRNAs. It was discovered that in ESCs, a significant number of lincRNA promoters carry H3K4me1 marks instead of having H3K4me3. Conversely, a large fraction of the Polycomb-regulated lincRNAs marked by bivalent domain undergoes $\mathrm{H} 3 \mathrm{~K} 27$ me3-mediated silencing upon ESC differentiation.

Which of the alleles is expressed may dependent on each allele's parental origin. Leung et al. [21] delineated allelic differences using a chromosome-spanning haplotype reconstruction strategy. Extensive allelic biases have been revealed in both chromatin modifications and transcriptome across different tissues and individuals. In the study by Farh et al. [22], the candidate causal variants for 21 autoimmune diseases have been identified from genotyping data. The causal variants tend to occur in close proximity to binding sites for signal-dependent TFs specific in immune response.

The role of epigenetic variability in the same cell type in the human population was clarified recently [23]. According to this research, there is increased variability of DNA methylation at candidate enhancers linked to genes that are at the transitional or 'poised' state. The reported findings support the idea that epigenetic heterogeneity between individuals is one of the major mechanisms to sustain cell-to-cell variability.

\section{Acknowledgement}

The author wants to thank D-G Shin for helpful discussions.

\section{Financial \& competing interests disclosure}

The author has no relevant affiliations or financial involvement with any organization or entity with a financial interest in or financial conflict with the subject matter or materials discussed in the manuscript. This includes employment, consultancies, honoraria, stock ownership or options, expert testimony, grants or patents received or pending, or royalties.

No writing assistance was utilized in the production of this manuscript. 


\section{References}

1 Romanoski CE, Glass CK, Stunnenberg HG et al. Epigenomics: roadmap for regulation. Nature 518(7539), 314-316 (2015).

2 Roadmap Epigenomics Consortium, Kundaje A, Meuleman W et al. Integrative analysis of 111 reference human epigenomes. Nature 518(7539), 317-330 (2015).

3 Siersbæk R, Rabiee A, Nielsen R et al. Transcription factor cooperativity in early adipogenic hotspots and superenhancers. Cell Rep. 7(5), 1443-1455 (2014).

4 Ziller MJ, Edri R, Yaffe Y et al. Dissecting neural differentiation regulatory networks through epigenetic footprinting. Nature 518(7539), 355-359 (2015).

5 Whyte WA, Orlando DA, Hnisz D et al. Master transcription factors and mediator establish super-enhancers at key cell identity genes. Cell 153(2), 307-319 (2013).

6 Hnisz D, Abraham BJ, Lee TI et al. Super-enhancers in the control of cell identity and disease. Cell 155(4), 934-947 (2013).

7 Siersbæk R, Baek S, Rabiee A et al. Molecular architecture of transcription factor hotspots in early adipogenesis. Cell Rep. 7(5), 1434-1442 (2014).

8 Tsankov AM, Gu H, Akopian V et al. Transcription factor binding dynamics during human ES cell differentiation. Nature 518(7539), 344-349 (2015).

9 Liu Z, Merkurjev D, Yang F et al. Enhancer activation requires trans-recruitment of a mega transcription factor complex. Cell 159(2), 358-373 (2014).

10 Xie X, Rigor P, Baldi P. MotifMap: a human genome-wide map of candidate regulatory motif sites. Bioinformatics 25(2), 167-174 (2009).

11 Heidari N, Phanstiel DH, He C et al. Genome-wide map of regulatory interactions in the human genome. Genome Res. 24(12), 1905-1917 (2014).

12 Schoenfelder S, Furlan-Magaril M, Mifsud B et al. The pluripotent regulatory circuitry connecting promoters to their long-range interacting elements. Genome Res. 25(4), 582-597 (2015).
13 Dixon JR, Selvaraj S, Yue F et al. Topological domains in mammalian genomes identified by analysis of chromatin interactions. Nature 485(7398), 376-380 (2012).

14 Rao SS, Huntley MH, Durand NC et al. A 3D map of the human genome at kilobase resolution reveals principles of chromatin looping. Cell 159(7), 1665-1680 (2014).

15 Dowen JM, Fan ZP, Hnisz D et al. Control of cell identity genes occurs in insulated neighborhoods in mammalian chromosomes. Cell 159(2), 374-387 (2014).

16 Vietri Rudan M, Barrington C, Henderson S et al. Comparative $\mathrm{Hi}-\mathrm{C}$ reveals that CTCF underlies evolution of chromosomal domain architecture. Cell Rep. 10(8), 1297-1309 (2015).

17 Cuadrado A, Remeseiro S, Graña O et al. The contribution of cohesin-SA1 to gene expression and chromatin architecture in two murine tissues. Nucleic Acids Res. 43(6), 3056-3067 (2015).

18 Dixon JR, Jung I, Selvaraj S et al. Chromatin architecture reorganization during stem cell differentiation. Nature 518(7539), 331-336 (2015).

19 Babaei S, Akhtar W, de Jong J et al. 3D hotspots of recurrent retroviral insertions reveal long-range interactions with cancer genes. Nat. Commun. 6, 6381 (2015).

20 Amin V, Harris RA, Onuchic V et al. Epigenomic footprints across 111 reference epigenomes reveal tissue-specific epigenetic regulation of lincRNAs. Nat. Commun. 6, 6370 (2015).

21 Leung D, Jung I, Rajagopal $\mathrm{N}$ et al. Integrative analysis of haplotype-resolved epigenomes across human tissues. Nature 518(7539), 350-354 (2015).

22 Farh KK, Marson A, Zhu J et al. Genetic and epigenetic fine mapping of causal autoimmune disease variants. Nature 518(7539), 337-343 (2015). Wijetunga NA, Delahaye F, Zhao YM et al. The meta-epigenomic structure of purified human stem cell populations is defined at cis-regulatory sequences. Nat. Commun. 5, 5195 (2014). 